\title{
Community-Based Interdisciplinary Research: Introduction to the Special Issue
}

\author{
Kenneth I. Maton • Douglas D. Perkins • \\ David G. Altman • Lorraine Gutierrez • \\ James G. Kelly · Julian Rappaport · Susan Saegert
}

Published online: 22 June 2006

(C) Springer Science+Business Media, Inc. 2006

\begin{abstract}
This special issue on community-based interdisciplinary research grew out of the work of the SCRA Interdisciplinary Task Force and an Interdisciplinary Working Conference held at Vanderbilt University in May, 2004. In this introduction to the special issue, the historical context for interdisciplinary underpinnings for community psychology theory, research, action and training is first depicted. This is followed by a brief description of the mission and work of the recent SCRA Interdisciplinary Task Force and the Interdisciplinary Working Conference. The introduction concludes with a brief summary of the papers in the two main sections of the special issue, Prospects and
\end{abstract}

The authors are members of the Society for Community Research and Action Interdisciplinary Task Force.

\author{
K. I. Maton $(\bowtie)$ \\ Department of Psychology, University of Maryland Baltimore \\ County, Baltimore, MD 21250 \\ e-mail: maton@umbc.edu \\ D. D. Perkins \\ Vanderbilt University \\ D. G. Altman \\ Center for Creative Leadership \\ L. Gutierrez \\ University of Michigan \\ J. G. Kelly \\ University of California, \\ Davis \\ J. Rappaport \\ University of Illinois at Urbana-Champaign \\ S. Saegert \\ City University of New York
}

Perspectives (four scholarly papers and three commentaries) and Community-Based Interdisciplinary Action-Research (four interdisciplinary action-research projects).

Keywords Interdisciplinary - Community psychology · Action-research · Transdisciplinary · Multidisciplinary . Collaboration $\cdot$ Collaborative research

Two of the most significant events in the history of community psychology in the United States were also ones with strong and explicit implications that the field must become more interdisciplinary. The first was the 1965 Conference on the Education of Psychologists for Community Mental Health held in Swampscott, MA (Anderson et al., 1966). ${ }^{1}$ A full chapter of the Swampscott report was devoted to relations with other disciplines and concluded:

The broadly interdisciplinary orientation of the Conference was most definitively reflected in its strong emphasis on the participation of many disciplines in the training of psychologists for community work. The consensus was that degree programs of training should involve a multidisciplinary faculty.

Many participants agreed that training would best be carried out with an interdisciplinary student body. Most often mentioned were: sociology, social work, medicine, anthropology, political science, education, public health, and economics. Other suggested disciplines were: nursing, law, business administration, city planning, philosophy, and theology.

\footnotetext{
${ }^{1}$ The conference title reflects its primary original agenda, but in titling the Swampscott conference report the authors used the bolder, newer, broader term "Community Psychology."
} 
... as one group put it, 'We would look with favor on any interdisciplinary arrangements that are possible.' (Anderson et al., 1966, p. 17)

Lest the reader infer that the above list only refers to the background of students in community psychology programs, the summary chapter makes clear that these are all fields that were recommended for close collaboration (p. 29).

The Swampscott report specifically identified "ecological psychology" research by Barker and colleagues as exemplary, a point echoed decades later by Shinn (1987) and others in prodding community psychology to expand its domain to research on prevention and empowerment in five types of behavior settings identified by Barker (1968): schools, work sites, religious settings, voluntary associations, and government. Such settings deal with what have been called "problems of the third kind," or ones that are temporally pressing, of practical or political significance, and inherently multidisciplinary (Klein, 2000), as opposed to intradisciplinary and multidisciplinary problems that are more purely intellectual in nature.

A second momentous event in the history of the field in the U.S. was the creation of the Society for Community Research and Action (SCRA) in 1989. There were several reasons for the creation of SCRA as a professional organization independent of the American Psychological Association (APA), some pragmatic and some substantive. The two substantive purposes for the change each had important interdisciplinary implications (Meritt, Greene, Jopp, \& Kelly, 1998). One was to lend greater recognition to the existing diversity of interests and methods within the field, in terms of both scientific and applied work and the fact that community psychologists view research and action as inextricably connected. The other central purpose of SCRA's creation was to attract new members from fields outside of psychology and to promote more interdisciplinary collaboration to better understand and address multifaceted social issues in all their contextual and theoretical complexity (Merritt et al., 1998).

Unlike mainstream psychology's predominant focus on the intrapsychic life and behavioral health of individuals, community psychology has focused on the development and study of interventions to ameliorate the lives of people in the many diverse settings in which they live, work, play, pray, go to school, and seek help. Moreover, community psychology explicitly directed its attention to individuals and to the context in which individuals lived their lives. The pioneers of community psychology recognized that the field had much to learn from all the social sciences and professional disciplines about how to design and evaluate prevention programs; consult with self-help groups, community organizations, schools, and public and private human service agencies; and analyze policies. We must engage in these applied activities with sensitivity and understanding about larger so- cial structures and dynamics, cultural diversity, political and economic considerations, and the social and physical environment.

The critical need for community psychology to become more interdisciplinary was thus well known to the founders of community psychology and has been repeatedly voiced in conferences, books, classrooms, intervention sites, and in the development of SCRA itself and its organizational structure. Research A few, isolated, interdisciplinary graduate programs have been developed (Reynolds, 1997), but given the great, early promises of interdisciplinary cross-fertilization, progress toward a fully interdisciplinary community psychology and SCRA has been surprisingly slow and difficult (due in part to the insularity of academic disciplines in general and of psychology departments in particular).

Why have so few interdisciplinary training programs in the field been developed and why do so few individuals from other disciplines become involved in SCRA? Why are exemplary interdisciplinary collaborative community research projects still so relatively rare (if co authorship of journal articles is any indication)? Why have we not made a more serious and systematic effort to reach out to and work with allied disciplines? Why have many funding agencies made it difficult to obtain funding for expansive interdisciplinary work? These and other questions are touched upon throughout this special issue, along with examples of successful interdisciplinary efforts and the various factors that facilitate vibrant and effective interdisciplinary work.

\section{Interdisciplinary task force and working conference}

These were just some of the questions and considerations that led to a concerted interdisciplinary agenda as part of Kenneth Maton's 1998-1999 SCRA Presidency (Maton, 2000), leading to the establishment of the SCRA Interdisciplinary Task Force in 2001. The current members of the Task Force are the co-editors of this special issue. The Task Force was charged with the mission of reviewing various mechanisms through which the interdisciplinarity of community psychology could be enhanced. Responding to this charge, the Task Force generated a list of various means to enhance the interdisciplinary focus of the field, encompassing research, training, action, and exchanges with like minded groups from other disciplines, and submitted it to the Executive Committee. One of the recommendations of the Task Force, establishment of a new standing SCRA committee focused on enhancing interdisciplinary linkages, was approved by the SCRA Executive Committee in 2003.

Early on, the Task Force decided that one important contribution it could make to enhance interdisciplinary collaboration in community-based research would be the sponsorship of a small, interactive conference. The conference would 
include leading interdisciplinary teams doing communitybased research, as well as junior and senior researchers in community psychology, the current and future opinion leaders in our field. Funding was obtained from The Robert Wood Johnson Foundation (and was later matched by the conference host, Vanderbilt University), and several interdisciplinary teams were selected to present their work. Surprisingly, the pool of exemplary interdisciplinary teams whose published work clearly depicted the value of bringing together multiple disciplines for community-based research was not large. Even smaller was the pool of research in which truly interdisciplinary theory, methods, and interventions were developed or implemented.

Vanderbilt University's Program in Community Research and Action was selected to host the conference. The Vanderbilt program had its own particular reason for hosting the conference, in addition to genuine interest in gaining new insights into interdisciplinary research and graduate education. Earlier, J.R. Newbrough had resurrected James G. Kelly's idea for a "Woods Hole"-style collaborative field station for community psychology, which evolved into the SCRA Community Action-Research Centers network (cf. Kelly, 1970). Newbrough served as the initial hub of the network and in 2004 Vanderbilt was developing its own new, interdisciplinary Center for Community Studies. ${ }^{2}$ The conference was seen as an opportunity to both mark the public launch of the Center and invite experts in community psychology and related fields from around the country to advise on its future direction.

The conference was held May 20-23, 2004, at Vanderbilt's Peabody College and was attended by 80 researchers. Half of the participants were from various parts of the U.S. and Canada, and the other half were Vanderbilt faculty and Ph.D. students (see Appendix for a list of conference participants). The conference goals were to help make clear: (1) the processes that lead to productive interdisciplinary research among research team members and between researchers and community members; (2) the promise of interdisciplinary research, including the breakthroughs in understanding about community-based phenomena and resulting communitybased action; and (3) the contextual factors that contribute to successful interdisciplinary research and the obstacles that inhibit it, each at multiple levels including the department, school or college, university, discipline, and community.

\footnotetext{
${ }^{2}$ The Center for Community Studies is a member of both the SCRA Community Action-Research Centers and an international network of research teams studying the organization and use of power in community contexts. The Center has established collaborative Work Groups in the areas of Healthy Communities, Schools and Community, Organizational Change, Urban Neighborhoods, International Communities, and Religion, Spirituality, and Community (see http://peabody.vanderbilt.edu/ccs/).
}

Presenters were asked to address each of these goals, as well as any of the following specific questions, based on their experience: (1) What lessons can be learned about creating a setting that facilitates true interdisciplinary work? (2) What are the benefits and costs of conducting this type of work? (3) What facilitates or impedes interdisciplinary work? (4) What factors motivate individuals to get involved in this work? (5) What impact did the interdisciplinary collaboration have on the questions asked, the methods used, the actions taken, and the impacts obtained? (6) What are the key systemic values that promote interdisciplinary collaboration? (7) What are some specific steps to reduce interdisciplinary conflicts and inequities? (8) How is the "community" of interest defined in the research; do different disciplines define this community differently; and what is the process used to come to a common understanding? (9) What kind of training and mentoring do doctoral students from different fields need to enable them to function in interdisciplinary teams?

The three featured interdisciplinary research teams, and a fourth presenter, each discussed the challenges, successes, and lessons of their collaborations. Their presentations, and the resulting discussion, indicated that although it is not necessarily easy, satisfying, manageable and useful communitybased interdisciplinary work is clearly feasible. One team included a medical anthropologist, an ecologist, and an executive director of a community-based agency (cf. Schensul et al., this issue). A second was composed of a faculty member from a school of architecture and a faculty member from a school of social work involved in an interdisciplinary center focused on environmental education and design (cf. Sutton \& Kemp, this issue). The third included an epidemiologist and a grass-roots community organizer (Syme, HendersonJames, \& Ritterman, 2004, May). The fourth presenter was a faculty member in social ecology, and lead evaluator examining the collaborative processes and outcomes emerging within a set of NIDA-funded transdisciplinary tobacco use research centers (cf. Stokols, this issue).

Aside from those prepared presentations, all participants at the conference shared personal stories and experiences related to interdisciplinary research highlights, challenges, and lessons learned. The conference format was highly participatory and led by a professional facilitation team using an "open space" design, in which substantial time was devoted to small breakout group discussions on emergent topics of mutual interest to participants. The ultimate purpose of the conference was for participants (both researchers and practitioners) to build connections, take away new ideas for interdisciplinary collaboration, apply them in their own work, and share those ideas with a larger audience-in part through this special issue. An invitation to submit papers for the special issue was made to all conference participants; all papers in the special issue, then, are authored by conference participants. 


\section{Overview of this issue}

The special issue is divided into two main sections. The first, Prospects and Perspectives, includes four scholarly papers and three commentaries that examine the prospects, current status, and future directions for interdisciplinary communitybased research and action. The first paper, by Kenneth Maton, Douglas Perkins and Susan Saegert, emphasizes the critical importance of and the current prospects for enhanced interdisciplinary cross-fertilization and collaboration in community psychology (Maton, Perkins, \& Saegert, this issue). Generic challenges to interdisciplinary work across disciplines, and challenges distinct to community psychology, are each presented. The latter include the difficulty of multilevel, integrative work that crosses higher levels of analysis, and the complexities involved in simultaneously bringing together multiple disciplinary collaborators and community partners. The authors delineate a set of facilitative factors necessary to overcome both the general and communitypsychology-specific barriers to interdisciplinary work, and outline a series of concrete steps the field can take to enhance the development of a vibrant interdisciplinary, multilevel discipline.

Three commentaries on the Maton, Perkins, and Saegert article follow. The first, by urban studies researcher Janet Smith, questions the authors' implicit predilection for transdisciplinary collaboration, in which the disciplinary perspectives of collaborators are merged, resulting in a new, integrative perspective (Smith, this issue). She describes the countervailing advantages, in her experience, of bringing to bear separate, distinct disciplinary perspectives on phenomena of interest. Community psychologist Beth Shinn, in her commentary, argues that the challenges to interdisciplinary research in the article are overstated, based on the ease in her experience of engaging in a diversity of interdisciplinary collaborations with like-minded colleagues from other disciplines who share substantive interests. She concludes her article with a caution concerning the costs of developing a more interdisciplinary identity for community psychology, especially for faculty and programs currently housed within psychology departments. Hiro Yoshikawa, also a community psychologist, agrees with the general framework presented in the article, and emphasizes the importance of identifying specific phenomena of interest that cross ecological levels in which community psychology has something to provide other disciplines, and those in which community psychology has something to learn (Yoshikawa, this issue). Implementation research, and the efficacy, effectiveness, adoption and replication of interventions are presented as examples of the former, and analysis of macro-level factors and distinctive methodologies as examples of the latter.

Heather Davidson and colleagues are situated in the disciplines of applied social psychology, community psychol- ogy, community research and action, counseling, critical psychology, higher education administration, program evaluation, quantitative methods, theology, and sociology. Their article provides an important case example, focused on issues of power and action, of what other disciplines have to offer community psychology, and what our field can offer them (Davidson et al., this issue). Specifically, they examine the implications for community psychology of the extent to which "critical scholarship" in other disciplines incorporates a focus on power and action, respectively. Applying a systematic method to review journals in six disciplines, they find that whereas community psychology is more action oriented than critical scholarship in these disciplines, the other disciplines are more likely to explicitly examine and challenge institutionalized power structures and the status quo in their scholarship. The authors conclude the article with a number of implications for the development of a more critical community psychology.

Is the challenge of collaborating across disciplines comparable to the challenge of interacting with persons from different cultures? Viewing disciplines as distinct cultural groups, Stephanie and Jennifer Reich (Reich \& Reich, this issue), representing the disciplines of community psychology and sociology, respectively, examine the implications of acquired wisdom in the field of cultural competence to the enhancement of interdisciplinary collaborations. Specifically, they propose that for interdisciplinary collaborations to be successful, participants must value disciplinary diversity, develop the capacity for self-assessment, work towards understanding one's own disciplinary culture, and be sensitive to the dynamics present when different cultures come into contact. They also stress the importance of developing increased awareness of the power dynamics at play when disciplines interact, and of avoiding tokenism, informal hierarchies and disciplinary policing.

The final paper in the first section, by Daniel Stokols, a faculty member in a school of social ecology, articulates an ambitious conceptual framework for transdisciplinary action research that encompasses a broad range of collaborative domains (Stokols, this issue). Specifically, the paper examines what is known about the challenges and facilitating contexts for three key types of action-research collaboration: 1) collaboration among scholars representing different disciplines; 2) collaboration among researchers from multiple fields and community practitioners representing diverse professional and lay perspectives, and 3) collaboration among community organizations across local, state, national and international levels. Towards the goal of establishing a science of transdisciplinary action research, Stokols emphasizes the need in the years ahead for expanded, explicit research focus on the processes, contexts and outcomes of each type of transdisciplinary action research collaboration. 
The second section in the special issue, Community-based Interdisciplinary Action Research, includes four examples of community-based projects initiated by interdisciplinary teams. The interdisciplinary projects vary greatly in content and method, and in the relative focus on research and action. They serve to depict representative examples of work in the action-research arena, and to illustrate some of the associated benefits, challenges, and facilitating factors of interdisciplinary collaboration work to date. Jean Schensul, a medical anthropologist situated in a free-standing community research institute, and colleagues developed an interdisciplinary/intersectoral action-research partnership to better understand the extent of depression and culturespecific issues of definition and barriers to care among impoverished older minority adults living in subsidized public and private housing. Multiple disciplines and community partners were involved (Schensul et al., this issue). A mixed research design, encompassing epidemiological and ethnographic methods along with action components, was jointly developed in the larger team context. Factors facilitating the successful interdisciplinary collaboration included shared values concerning the issue, a prior history of working together, joint conceptualization of the research, strong management skills to negotiate issues that emerged, inclusion of all research team members in research decision making, a balancing of research and service goals, and political will to advocate for increased focus on health inequities.

Stephen Schensul, an anthropologist and faculty member in a school of medicine, and colleagues conducted an international, transdisciplinary and intersectoral collaboration in their large scale, multi-year HIV/STD prevention project focused on married men in an urban poor community in Mumbai (Bombay), India (Schensul, Nastasi, \& Verma, this issue). The research revealed gender and culture-specific underpinnings of AIDS transmission and prevention, and generated a unique intervention model involving both traditional and non-traditional local resources. Challenges to collaboration included geographic distance, institutional culture, language differences, and personal and disciplinary differences in perspectives and practices. Factors facilitating successful collaboration included the development of close personal relationships between core collaborators in cultural context, regular presence on site in India, effective use of electronic communication, reaching agreement on a transdisciplinary model, shared values related to project goals, long-term professional relationships, and openness of team members to learn from each other.

Yolanda Suarez-Balcazar, a community psychology faculty member and colleagues from four academic institutions and three community-based organizations joined together to address the issue of access to health foods and nutrition in a working class African American neighborhood in Chicago (Suarez-Balcazar et al., this issue). The disciplines of community development and community organizing, community psychology, geography, nursing, nutrition, public health, sociology and urban planning and policy were represented, each contributing to one or more partnership projects. Assets-based community development theory, cognitive-behavioral theory, and/or participatory evaluation approaches guided work on understanding and enhancing children's diets and food options in the local school, access to healthy food in local stores, and evaluation of a local summer farmer's market. Factors facilitating the success of the collaboration included strong levels of commitment among members to: addressing the issue, sharing research methods, working together, sharing partnership resources, cultural competency, and sharing power.

Sharon Sutton and Susan Kemp, faculty members in schools of architecture and social work, respectively, in their article describe the use of interdisciplinary design charrettes as a tool for community problem-solving (Sutton \& Kemp, this issue). Three charrettes are described, each involving design and social science faculty and students, and community members. The charrettes bring together usually disparate visual and analytical inquiry tools, and create "a space apart" for community members to engage in co-learning that helps them to further understand local issues and consider novel solutions. Challenges faced include difficulties in balancing the design and social science disciplines, the fact that the social scientists felt "out of place" on the designers' home turf, and interpersonal conflicts arising in some charrettes grounded in ethnic group differences. Benefits of the collaboration included expanded knowledge resulting from multiple modes of inquiry, and bringing to bear complementary skills (i.e., visual; analytical; interpersonal) from members of the different disciplines in the design and community interaction facets of the charrettes.

This special issue represents an initial foray into the exciting and important domain of interdisciplinary collaboration. Grounded in the historical vision of the founders of community psychology, interdisciplinary cross-fertilization in theory, research, action and training is essential for the vibrancy and effectiveness of our field. The current papers provide a snapshot of current perspectives and practices in the interdisciplinary arena. The challenges, positive potential, and feasibility of interdisciplinary work are depicted throughout the issue. Our hope is that it will stimulate increased attention, reflection, dialogue and action to enhance the interdisciplinary nature of our field, including an ongoing effort to identify, learn from and build upon exemplary models of interdisciplinary collaboration that cross multiple levels, disciplines, and community sectors. In this way, we can enhance our capacity to make a sustained difference in the lives of our citizens, their communities, and our larger society. 


\section{Appendix: Conference Participants}

Sherry Ahrentzen (Synthesis Team), Architecture, University of Wisconsin-Milwaukee

Nicole Allen, Psychology, University of Illinois-Urbana-Champaign

David Altman (Synthesis Team), Center for Creative Leadership, Greensboro, NC

Theresa Armstead, Community Research \& Action, Vanderbilt University

Rhonda BeLue, Health Policy \& Administration, Pennsylvania State University

Mary Berlin (Lead Facilitator), Berlin \& Co.

Kimberly Bess, Community Research \& Action, Vanderbilt University

Susan Brooks, Law School, Vanderbilt University

Barbara B. Brown, Family \& Consumer Studies, University of Utah

Vera Chatman, Community Research \& Action, Vanderbilt University

Daniel Cooper, Community Research \& Action, Vanderbilt University

Melinda Coston, Community Research \& Action, Vanderbilt University

Joseph Cunningham, Community Research \& Action, Vanderbilt University

Heather Davidson, Psychology \& Human Development, Peabody College, Vanderbilt University

Marsha Davis, Community Research \& Action, Vanderbilt University

Paul Dokecki, Community Research \& Action, Vanderbilt University

Scot Evans, Community Research \& Action, Vanderbilt University

Gina Frieden, Human Development Counseling, Vanderbilt University

Dennis Gorman (Synthesis Team), School of Rural Public Health, Texas A\&M University

Lorraine Gutierrez (Synthesis Team), Social Work, University of Michigan

Carrie Hanlin, Community Research \& Action, Vanderbilt University

Gary Harper, Psychology, DePaul University

Craig Anne Heflinger, Community Research \& Action, Vanderbilt University

Nathan Henderson-James (Presenter), Association of Communities Organized for Reform Now, California

Cheri Hoffman, Community Research \& Action, Vanderbilt University

Sarah Van Hooser, Community Research \& Action, Vanderbilt University

Joseph Hughey, Psychology, University of Missouri-Kansas City

Keith Humphries, Center for Health Care Evaluation, Stanford University

Ron Hustedde (Synthesis Team), Rural Sociology, University of Kentucky

Lynette Jacobs-Priebe, Community Research \& Action, Vanderbilt University

Diana Jones, Community Research \& Action, Vanderbilt University

Susan Kemp (Presenter), School of Social Work, University of Washington, Seattle

Christopher Keys, Psychology, DePaul University

Donald Klein, Union Institute and University

Murray Levine, Psychology, State University of New York at Buffalo (Emeritus)

Daniel Lewis, Inst. for Policy Research, Dept. of Education \& Social Policy, Northwestern University

D. Adam Long, Psychology, A\&S, Vanderbilt University

Melanie Lutenbacher, Nursing, Vanderbilt University

Eric Mankowski, Psychology, Portland State University

Kenneth Maton (Co-Chair, Synthesis Team), Psychology, University of Maryland-Baltimore County

Robin Miller, Psychology, Michigan State University

Lynne Mock, Psychiatry, University of Illinois-Chicago

Maury Nation, Human Development Counseling, Community Research \& Action, Vanderbilt University

Geoff Nelson, Psychology, Wilfrid Laurier University

J.R. Newbrough (Co-facilitator), Community Research \& Action, Vanderbilt University

William Partridge, Community Research \& Action, Vanderbilt University

Allison Patten Maguire, Psychology \& Human Development, Peabody College, Vanderbilt University

Douglas D. Perkins (Co-Chair, Synthesis Team/Chair, Host Cmte.), Community Research \& Action, Vanderbilt University

Sheila Peters, Psychology, Fisk University

Tracy Pinkard, Psychology \& Human Development, Peabody College, Vanderbilt University

Isaac Prilleltensky, Community Research \& Action, Vanderbilt University

Ora Prilleltensky, Human Development Counseling, Human \& Org. Development, Vanderbilt University

Matthew Ramsey, History; Center for Medicine, Health \& Society, Vanderbilt University

Julian Rappaport (Synthesis Team), Psychology, University of Illinois-Urbana-Champaign

Peter Redvers-Lee, Community Research \& Action, Vanderbilt University

Stephanie Reich, Psychology \& Human Development, Peabody College, Vanderbilt University 


\section{Appendix: Continued}

Tracey Revenson, Psychology, City University of New York Graduate Center

Carmen Reyes (Presenter), North Central Area Agency on Aging, Connecticut

Kelly Richardson, Community Research \& Action, Vanderbilt University

Manuel Riemer, Psychology \& Human Development, Peabody College, Vanderbilt University

Stephanie Riger, Psychology and Gender \& Women's Studies, University of Illinois-Chicago

Julie Robison (Presenter), Braceland Ctr for Mental Health \& Aging, Institute of Living, Hartford Hospital

Susan Saegert (Synthesis Team), Psychology, City University of New York Graduate Center

Lori Schnieders, Human Development Counseling, Community Research \& Action, Vanderbilt University

Jean J. Schensul (Presenter), Institute for Community Research, Hartford, CT

Steven Schensul (Sunday co-facilitator), Center for International Community Health Studies, University of Connecticut

Irma Serrano-Garcia, Psychology, University of Puerto Rico

Sharon Shields, Human \& Organizational Development, Vanderbilt University

Marybeth Shinn, Psychology/Wagner Graduate School of Public Service, New York University

Andrew Shookhoff, Child \& Family Policy Center, Vanderbilt Inst. for Public Policy Studies

Tod Sloan (Co-Facilitator), Psychologists for Social Responsibility, Counseling Psychology, Lewis \& Clark College

Janet Smith, Urban Studies/Planning, University Illinois-Chicago, Urban Affairs Association Board

Paul Speer, Community Research \& Action, Vanderbilt University

Dan Stokols (Presenter), School of Social Ecology, University of California-Irvine

Yolanda Suarez (CA-RC Co-chair), Psychology, University Illinois-Chicago

Sharon Sutton (Presenter), School of Architecture, University of Washington, Seattle

S. Leonard Syme (Presenter), Public Health \& Epidemiology, UC-Berkeley (Emeritus)

Sarah Van Hooser, Community Research \& Action, Vanderbilt University

Kenneth Wallston, Nursing, Vanderbilt University

Hirokazu Yoshikawa, Graduate School of Education, Harvard University

Marc Zimmerman, Public Health, University of Michigan

\section{References}

Anderson, L. S., Cooper, S., Hassol, L., Klein, D. C., Rosenblum, G., \& Bennett, C. C. (1966). Community psychology: A report of the Boston Conference on the Education of Psychologists for Community Mental Health. Boston, MA: Boston University \& Quincy Mass. South Shore Mental Health Center.

Barker, R. G. (1968). Ecological psychology: Concepts and methods for studying the environment of human behavior. Stanford, CA: Stanford University Press.

Davidson, H., Evans, S., Ganote, C., Hernickson, J., Jacobs-Priebe, L., Jones, D. L., Prilleltensky, I., \& Riemer, M. (this issue). Power and action in critical theory across disciplines: Implications for critical community psychology.

Kelly, J. G. (1970). Antidote for arrogance: Training for community psychology. American Psychologist, 25, 524-531.

Klein, J. T. (2000). A conceptual vocabulary of interdisciplinary science. In P. Weingart \& N. Stehr (Eds.), Practising interdisciplinarity (pp. 3-24). Toronto: University of Toronto Press.

Maton, K. I. (2000). Making a difference: The social ecology of social transformation. American Journal of Community Psychology, 28(1), 25-57.

Maton, K. I., Perkins, D. D., \& Saegert, S. (this issue). Community psychology at the crossroads: Prospects for interdisciplinary research.

Meritt, D. M., Greene, G. J., Jopp, D. A., \& Kelly, J. G. (1998). A history of Division 27 (Society for Community Research and Action). In D. A. Dewsbury (Ed.), Unification through division: Histories of the divisions of the American Psychological Association (Vol. 3, pp. 73-99). Washington, DC: American Psychological Association.

Reich, S., \& Reich, J. (this issue). Cultural competence in interdisciplinary collaborations: A method for respecting diversity in research partnerships.
Reynolds, A. J. (1997). Interdisciplinary programs in community and applied research. Journal of Prevention and Intervention in the Community, 15(1), 65-82.

Schensul, J. J., Robison, J., Reyes, C., Radda, K., Gaztambide, S., \& Disch, W. (this issue). Building interdisciplinary/intersectoral research partnerships for community-based mental health research with older minority adults.

Schensul, S. L., Nastasi, B. K., \& Verma, R. K. (this issue). Communitybased research in India: A case example of international and transdisciplinary collaboration.

Shinn, M. (1987). Expanding community psychology's domain. American Journal of Community Psychology, 15(5), 555-574.

Shinn, M. (this issue). External, not internal challenges to interdisciplinary research.

Smith, J. L. (this issue). At the crossroad: Standing still and moving forward.

Stokols, D. (this issue). Toward a science of transdisciplinary action research.

Suarez-Balcazar, Y., Hellwig, M., Kouba, J., Redmond, L., Martinez, L., Block, D., Kohrman, C., \& Peterman, W. (this issue). The making of an interdisciplinary partnership: The case of the Chicago Food System Collaborative.

Sutton, S. E., \& Kemp, S. P. (this issue). Integrating social science and design inquiry through interdisciplinary design charrettes: An approach to participatory community problem solving.

Syme, S. L., Henderson-James, N., \& Ritterman, M. L. (2004, May). Public health has messages, people have lives: An effort to bridge the gap. Paper presented at the Society for Community Research and Action Working Conference on Interdisciplinary Collaboration, Vanderbilt University, Nashville, TN.

Yoshikawa, H. (this issue). Placing community psychology in the context of the social, health and educational sciences: Directions for interdisciplinary research and action. 